\title{
Variants of SARS-Cov-2: a cause of concern
}

\begin{abstract}
SARS-Cov-2 has emerged as one of the dreaded viruses that the world has so far faced. Ever since its outbreak, the virus has been baffling scientists with its unique inimitable pattern of mutation which leads to the beginning of several variants. Consequently, several parts of the world have gradually come under the grip of variants such as alpha, beta, gamma, delta etc. All these variants possess different transmission rate, mortality rate. Amid these variants, delta variant has emerged out to be highly transmissible leading to imposition of constraints in various sectors. This brief communication appraises reader about the variants of this virus, with special emphasis on delta variant. The transmissivity as well as infectivity rate of the variants are delineated in this brief article. In a comparative footing, the variants are highlighted along with plausible recommendations.
\end{abstract}

Volume 9 Issue 4 - 202I

\section{Biswas R}

Department of Physics, Tezpur University, India

Correspondence: Biswas R, Department of Physics, Applied optics and photonics lab, Tezpur University, Tezpur-784028, India, Tel 9954313970,Email rajib@tezu.ernet.in

Received: September 26, 2021 | Published: October 12, 2021

\section{Opinion}

Since 2020, the world has come under the grip of Covid-19 pandemic. First detected in Wuhan, China, the virus then gradually engulfed the whole world. The fatalities have so far been really agonizing. Around five million people had succumbed to Covid-19 so far. All nations including developed as well as developing has been reeling under the pressure of effective tackling of SARS-Cov-2. There has been second wave or third wave of this in many nations. Owing to unique mutation characteristics, this virus has transgressed into many variants which has become a serious concern for researchers, health policy makers as well as for the administration too. Consequently, many nations have reported detection of couple of variants such as alpha, beta, gamma, delta etc. These variants exhibit different properties. Notably, the transmission rate, mortality rate as well as infectivity rate need special mention here. These variants show different degree of transmissibility which thereby induce varying disease severity. By this, we refer to augmented hospitalizations or deaths. In addition, immunity developed through previous infections via formation of antibodies seems to be affected by these variants. Consequently, this triggers less efficacy in treatment as well as diagnosis. Among the reported variants, it has been found that the delta variant possesses highest transmissibility rate which incurs widespread infection among the populace. Accordingly, this brief opinion article appraises the variants of SARS-Cov-2 with special stress on delta variant. The characteristics such as transmissibility, viriality etc. of this variant has been concisely overviewed.

So far, as per report, Delta variant is $60 \%$ more contagious than other variants. It is more infectious than the original SARS-Cov-2 virus. ${ }^{1-5}$ Though a computer simulation model, researchers carried out the search the viriality of this variant. Furthermore, it was found that it possesses the capability of escaping immunity from prior infection half of time. Relatively, beta and gamma variants are found to be less transmissible. However, they outsmart in their bid to escape immunity. With extension to other variants, iota has been found to more detrimental to older generation. There have been generation of computer models to investigate the ability of transmissibility as well as immunity elusion. The models were based on mortality data from their epicenter. Noticeably, the newer variants emerge to be more widespread. ${ }^{4,5}$ Still, the available vaccines worldwide are well capable of impeding the growth of these variants. Mass vaccination can help in constraining the emergence of these new variants and thereby bringing an end to the Covid-19 epidemic. As for example, there has been drastic data decline in Covid 19 infections in India. This can be attributed to the implementation of public health measures. The researchers estimate that the Delta variant (B.1.617.2) is $10-20 \%$ more transmissible as compared to alpha type. As per data, in half of the individuals previously infected by the ancestral strain, the delta is found to evade prior immunity. ${ }^{6-8}$ Previous reports with data stemming from UK hint that Alpha is 1.5 time effective in causing infection. Upon comparing with Beta and gamma, it is found that Delta is highly transmissible; however less prudent in escaping the immunity as compared to the other two variants. Alpha is $50 \%$ more transmissible in comparison to the original SARS-Cov-2 virus; however, it possesses less ability to escape protection provided by prior infection. Another variant Iota (B.1.526) is computed to be $15-25 \%$ more transmissible. The most striking characteristic of this variant is that they pose immediate threat to the fatality compared to the pre-existing variants among older adults. ${ }^{9-12}$ Even though these variants seem to be dominating each other, however, the better way to prevent is the impeding of transmission. As per experts, mass immunization is one of the best strategies to deal with this pandemic. Furthermore, wearing of masks as well as maintaining distance can be the best solution for this. If there is strict adherence to covid appropriate behavior, there will be fewer chance of transmission which will incur direct decline in generation of more variants of SARS-Cov-2 to surface. However, only time will say.

\section{Acknowledgments}

There is no one to acknowledge.

\section{Conflicts of interest}

Author declared no conflict in interest.

\section{References}

1. Biswas R. Covid-19 and race for rapid diagnosis. J Bacteriol Mycol Open Access. 2021;9(2):54-55.

2. Biswas R. Nanosponges: a viable option for combating Covid-19. $J$ Clinical Research and Reports. 2020;5(4).

3. Biswas R. Are Men More Vulnerable to Covid-19 as Compared to Women? BioMed J Sci\& Tech Res. 2020;27(2).

4. Biswas R. An Application Overview of IoT Enabled-Big Data Analytics in Health Sector with Special Reference to Covid-19. Preprints. 2021:2021020203. 
5. Domingo E, Holland JJ. RNA virus mutations and fitness for survival. Annu Rev Microbiol. 1997;51:151-178.

6. Plante JA, Liu Y, Liu J, et al. Spike mutation D614G alters SARS-CoV-2 fitness. Nature. 592 (2021):116-121.

7. Baric RS. Emergence of a highly fit SARS-CoV-2 variant. $N$ Engl J Med. 2020;383:2684-2686.

8. Elbe S, Buckland-Merrett G. Data disease and diplomacy: GISAID's innovative contribution to global health. Glob Chall. 2017;1:33-46.

9. Korber B, Fischer WM, Gnanakaran S, et al. Tracking changes in SARSCoV-2 spike: evidence that D614G increases infectivity of the COVID-19 virus. Cell. 2020;182:812-827.
10. Kannan SR, Spratt AN, Quinn TP, et al. Infectivity of SARS-CoV-2: there is something more than D614G? J Neuroimmune Pharmacol. 2020;15:574-577.

11. Spratt AN, Kannan SR, Woods LT, et al. Evolution, correlation, structural impact and dynamics of emerging SARS-CoV-2 variants. Comput Struct Biotechnol J. 2021;19:3799-3809.

12. Kannan SR, Spratt AN, Cohen AR, et al. Evolutionary analysis of the Delta and Delta Plus variants of the SARS-CoV-2 viruses. Journal of Autoimmunity. 2021;124:102715. 